the present purpose to inquire), while all other sensations, as of hearing, smell, and taste, come before us only discontinuously and intermittently, not being had from all things nor always from the same things. But in a dog's experience touch cannot possibly co-operate with sight as it regularly does in ours. The organ of effective touch in man-touch that gets associated with vision -is in the last resort the hand, combining mobility and sensitiveness in the highest degree; and the $\mathrm{dog}$ has no hand. Its mobile limbs are not sensitive at the extremities, and, though it has sensitive lips, these, having no such active mobility as the human hand has, are extremely limited in the scope of their ap. prehension. Its touch being thus defective, what is there then in the dog to play second to sight, which as leader needs support, were it only because there is not always light to see with? Smell, I cannot but think, seeing that, while the organ is incontestably acute, it has the great advantage over the tactile surface of the lips, of receiving impressions from things already at a distance. If we only suppose-what the facts make very likely-that the dog's smell is acute enough to have some sen-ation from all bodies without exception, nothing more is wanting to enable a psychologist to understand that the dog's world may be in the main a world of sights and sinells continuous in space. In that case a dog conveyed in a basket might by smell alone find its way back pretty much as a man blindfolded finds his way by touch alone.

To argue properly so difficult a question is impossible in a short letter, and I must be content now, for reasons like those indicated rather than stated above, with giving my adhesion to $\mathrm{Mr}$. Wallace's view-so far at least as dogs are concerned, and to the extent that in smell we have a source of explanation for the phenomena which has never been sufficiently considered. That the explanation covers all the facts related even about dogs is more than I would assert; and whether it is equally serviceable for other animals like cats and horses, concerning which not less wonderful stories are told, is not so clear. Cats, however, seem to have very acute smell. What is the truth about the smell of horses?

University College, Feb. 24

\section{Fiords and Glacial Action}

In NATURE, vol. vii. pp. 94, 95, I find the following :-

"Pogoendorf's Annalen- $\Lambda$. Helland adduces a large amount of evidence to show that the fiords in Norway have been formed by glacial action."

It appears an obvious remark, and yet I have not met with it, that fiords are chiefly found in those coasts where from the geographical conditions there must have been the most glacial action. The most favourable conditicn; for glacial action are evidently those of a mountainous coast in a high and therefore cold latitude, fronting the rain and snow-laden west winds of the higher latitudes as they blow in from the ocean. These conditions are fulfilled in the highest degree by the coasts of Norway and Western Scotland: the western coast of North America from Vancouver's Island northwards; and the western coast of South America from Chilve southwards; and these coasts are accordingly more cut up into fiords than any others in the world.

The western coast of America along the enormously long lina from Vancouver's Is'and to Chiloe is one of the most unbroken in the world. It is significant that the change in the coast at Chiloe from an unbroken one to one very much broken into fiords is accompanied by a great and comparatively abrupt change in the height of perpetual snow on the Andes. The following are the heights of perpetual snow at three different latitudes, according to Mrs. Somerville's " Physical Geograp'y." The first two are north of Chiloe, the third south of it.

$$
\begin{aligned}
& \text { About } 33^{\circ} \mathrm{S} \text {. (near Valparaiso) . . . 12,780 feet }
\end{aligned}
$$

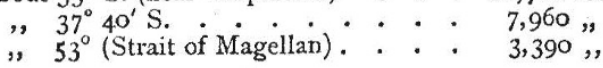

Although the height of the snow-line deperids chiefly on latitude, it is sensibly influenced by the aspect of the mountains respecting the rain and snow-bearing winds. The best instance of this is probably that of the Himalayas, where, according to Mrs. Somerville (page 3I4), the height of the snow-line is 16,620 feet on the noth side, and only 12,980 on the south. According to another anthority (Capt. Stuahey), qucted by Mrs. Somerville (p. 54), the heights are 19,000 to 20,000 feet on the north side, and $15,5 \mathrm{co}$ on the southern. The difference of the two estimates is about the same. The reason of the difference is evidently that the south side receives the moistureladen winds from the Indian Ocean. Old Forge, Dunmurry Joseph JohN MURPhY

\section{NOTE ON A POLYDACTYLOUS CAT FROM COOKHAM-DEAN}

$\mathrm{BY}$ the kindness of Dr. Piumbe, of Maidenhead, I have been able to procure one of these cats; and from the many curious points he possesses, I think a note on his peculiarities will interest some of the readers of NATURE.

Readers of Mr. Darwin's "Origin of Species" are familiar enough with the illustration he gives of correlation of arrest of development in the deafness of blue. eyed cats. Some years ago I showed that our great naturalist had fallen into error on this point, and that the correlation is not between the blue eyes and the deafness, but between the latter and the sex of the cat.

I have made a great many inquiries on this point, and have completely confirmed my former observation, that all perfectly white tom-cats are deaf, and that they have blue eyes occasionally, because that item of beauty is common among white cats. I have seen many white Tabithas with blue eyes, but none of them were deaf. My little "Pudge" from Cookham is perfectly deaf, and has one blue eye and a yellow one. For the first few days after I had him, I thought he could hear a little, but am now quite satisfied that his deafness is complete, though he is alive to sounds conveyed through solid media. A further point of interest is that he is rot mute as most deafs are, but there is a kittenish shrillness in his voice and a loudness in his purring, which are not commensurate with his age. I think, therefore, that it is possible that early in life he may have heard a little, for $I$ know of two instances where perfect mutism accompanied the deafness in cats, and I do not know of any contrary condition. The one yellow eye favours my view that "Putge" may have heard in infancy his mother's voice. His sense of touch is extremely acute compared to that of another cat I have, but his sight does not seem so sharp as that of cats generally is. He has twenty-six digits, and these are arranged-seven on each fore limb, and six on each hind limb. The supernumerary digits on the fore limbs are thumbs, and are placed one on either si.e of the true pollex, being joined to it, but having no metacarpal bones. In the hind limb the supernumerary digit is probably of the same nature, or a supernumerary index, being placed on the outer side of the hallux, and attached to the tarsus by a completely-developed metatarsal bone. LAWSON TAIT

\section{ON ACTION AT A DISTANCE*}

I HAVE no new discovery to bring before you this evening. I must ask you to go over very old ground, and to turn your attention to a question which has been raised again and again ever since men began to think.

The question is that of the transmission of force. We see that bodies at a distance from each other exert a mutual influence on each other's motion. Does thi.s mutual action depend on the existence of some third thing, some medium of communication, occupying the space between the bodies, or do the bodies act on each other immediately without the intervention of anjthing else?

The mode in which Faraday was accustomed to look at phenomena of this kind differs from that adopted by many other modern inquirers, and my special aim will be to enable you to place yourselves at Faraday's point of view, and to point out the scientific value of that con-

* Lecture delivered at the Royal Institution, Feb. 21, 1873, by Prot. Clerk Maxwell. 
ception of lines of force which, in his hands, became the key to the science of electricity.

When we observe one body acting on another at a distance, before we assume that this action is direct and immediate, we generally inquire whether there is any material connection between the two bodies, and if we find strings, or rods, or mechanism of any kind, capable of accounting for the observed action between the bodies, we prefer to explain the action by means of these intermediate connections, rather than to admit the notion of direct action at a distance.

Thus when we ring a bell by means of a wire, the successive parts of the wire are first tightened and then moved, till at last the bell is rung at a distance by a process in which all the intermediate particles of the wire have taken part one after the other. We may ring a bell at a distance in other ways, as by forcing air into a long tube at the other end of which is a cylinder with a piston which is made to fly out and strike the bell. We may also use a wire, but instead of pulling it, we may connect it at one end with a voltaic battery, and at the other with an electro-magnet, and thus ring the bell by electricity.

Here are three different ways of ringing a bell. They all agree, however, in the circumstance that between the ringer and the bell there is an unbroken line of communication, and that at every point of this line some physical process goes on by which the action is transmitted from one end to the other. The process of transmission is not instantaneous, but gradual, so that there is an interval of time after the impulse has been given to one extremity of the line of communication, during which the impulse is on its way, but has not reached the other end.

It is clear, therefore, that in many cases the action between bodies at a distance may be accounted for by a series of actions between each successive pair of a series of bodies which occupy the intermediate space, and it is asked, by the advocates of mediate action, whether, in those cases in which we cannot perceive the intermediate agency, it is not more philosophical to admit the existence of a medium which we cannot at present perceive, than to assert that a body can act at a place where it is not.

To a person ignorant of the properties of air, the transmission of force by means of that invisible medium would appear as unaccountable as any other example of action at a distance, and yet in this case we can explain the whole process, and determine the rate at which the action is passed on from one portion to another of the medium.

Why then should we not admit that the familiar mode of communicating motion by pushing and pulling with our hands is the type and exemplification of all action between bodies, even in cases in which we can observe nothing between the bodies which appears to take part in the action?

Here for instance is a kind of attraction with which Prof. Guthrie has made us familiar. A disc is set in vibration, and is then brought near a light suspended body, which immediately begins to move towards the disc as if drawn towards it by an invisible cord. What is this cord ? Sir W. Thomson has pointed out that in a moving fluid the pressure is least where the velocity is greatest. The velocity of the vibratory motion of the air is greatest nearest the disc. Hence the pressure of the air on the suspended body is less on the side nearest the disc than on the opposite side, the body yields to the greater pressure, and moves toward the disc.

The disc, therefore, does not act where it is not. It sets the air next it in motion by pushing it, this motion is communicated to more and more distant portions of the air in turn, and thus the pressures on opposite sides of the suspended body are rendered unequal, and it moves towards the disc in consequence of the excess of pressure.
The force is therefore a force of the old school-a case of vis à tergo, a shove from behind.

The advocates of the doctrine of action at a distance, however, have not been put to silence by such arguments. What right, say they, have we to assert that a body cannot act where it is not? Do we not see an instance of action at a distance in the case of a magnet, which acts on another magnet not only at a distance, but with the most complete indifference to the nature of the matter which occupies the intervening space? If the action depends on something occupying the space between the two magnets, it cannot surely be a matter of indifference whether this space is filled with air or not, or whether wood, glass, or copper, be placed between the maonets.

Besides this, Newton's law of gravitation, which every astronomical observation only tends to establish more firmly, asserts not only that the heavenly bodies act on one another across immense intervals of space, but that two portions of matter, the one buried a thousand miles deep in the interior of the earth, and the other a hundred thousand miles deep in the body of the sun, act on one another with exactly the same force as if the strata beneath which each is buried had been non-existent If any medium takes part in transmitting this action, it must surely make some difference whether the space between the bodies contains nothing but this medium, or whether it is occupied by the dense matter of the earth or of the sun.

But the advocates of direct action at a distance are not content with instances of this kind, in which the phenomena, even at first sight, appear to favour their doctrine. They push their operations into the enemy's camp, and maintain that even when the action is apparently the pressure of contiguous portions of matter, the contiguity is only apparent - that a space always intervenes between the bodies which act on each other. They assert, in short, that so far from action at a distance being impossible, it is the only kind of action which ever occurs, and that the favourite old vis a tergo of the schools has no existence in nature, and exists oniy in the imagination of schoolmen.

The best way to prove that when one body pushes another it does not touch it, is to measure the distance between them. Here are two glass lenses, one of which is pressed against the other by means of a weight. By means of the electric light we may obtain on the screen an image of the place where the one lens presses against the other. A series of coloured rings is formed on the screen. These rings were first observed and first explained by Newton. The particular colour of any ring depends on the distance between the surfaces of the pieces of glass. Newton formed a table of the colours corresponding to different distances, so that by comparing the colour of any ring with Newton's table, we may ascertain the distances between the surfaces at that ring. The colours are arranged in rings because the surfaces are spherical, and therefore the interval between the surfaces depends on the distance from the line joining the centres of the spheres. The central spot of the rings indicates the place where the lenses are nearest together, and each successive ring corresponds to an increase of about the 4,00oth part of a millimetre in the distance of the surfaces.

The lenses are now pressed together with a force equal to the weight of an ounce, but there is still a measurable interval between them, even at the place where they are nearest together. They are not in optical contact. To prove this, I apply a greater weight. A new colour appears at the central spot, and the diameters of all the rings increase. This shows that the surfaces are now nearer than at first, but they are not yet in optical contact, for if they were, the central spot would be black. I therefore increase the weights, so as to press the lenses into optical contact. But what we call optical contact is not real contact. 
Optical contact indicates only that the distance between the surfaces is much less than a wave-length of light. To show that the surfaces are not in real contact, I remove the weights. The rings contract, and several of them vanish at the centre. Now it is possible to bring two pieces of glass so close together, that they will not tend to separate at all, but adhere together so firmly that when torn asunder the glass will break, not at the surface of contact, but at some other place. The glasses must now be many degrees nearer than when in mere optical contact.

Thus ne have shown that bodies begin to press against each other while still at a measurable distance, and that even when pressed together with great force they are not in absolute contact, but may be brought nearer still, and that by many degrees.

Why, then, say the advocates of direct action, should we continue to maintain a doctrine founded only on the rough experience of a pre-scientific age, that matter cannot act where it is not, instead of admitting that all the facts from which our ancestors concluded that contact is essential to action were in reality cases of action at a distance, the distance being too small to be measured by their imperfect means of observation?

If we are ever to discover the laws of nature, we must do so by obtaining the most accurate acquaintance with the facts of nature, and not by ciressing up in philosophical language the loose opinions of men who had no knowledge of the facts which throw most light on these laws.

And as for those who introduce xtherial, or other media, to account for these actions, without any direct evidence of the existence of such media, or any clear understanding of how the media do their work, and who fill all space three and four times over with athers of different sorts, why the less these men talk about their philosophical scruples about admitting action at a distance the better:

If the progress of science were regulated by Newton's first law of motion, it would be easy to cultivate opinions in advance of the age. We should only have to compare the science of to-day with that of fifty years ago, and by producing, in the geometrical sense, the line of progress, we should obtain the science of fifty years hence.

The progress of science in Newton's time consisted in getting rid of the celestial machinery with which generations of astronomers had encumbered the heavens, and thus "sweeping cobwebs off the sky."

Though the planets had already got rid of their crystal spheres, they were still swimming in the vortices of Descartes. Magnets were surrounded by effluvia, and electrified bodies by atmospheres, the properties of which resembled in no respect those of ordinary effluvia and atmospheres.

When Newton demonstrated that the force which acts on each of the heavenly bodies depends on its relative position with respect to the other bodies, the new theory met with violent opposition from the advanced philosophers of the day, who described the doctrine of gravitation as a return to the exploded method of explaining everything by occult causes, attractive virtues, and the like.

Newton himself, with that wise moderation which is characteristic of all his speculations, answered that he made no pretence of explaining the mechanism by which the heavenly bodies act on each other. To determine the mode in which their mutual action depends on their relative position was a great step in science, and this step Newton asserted that he had made. To explain the process by which this action is effected was a quite distinct step, and this step, Newton, in his "Principia," does not attempt to make.

But so far was Newton from asserting that bodies really do act on one another at a distance, independently of any- thing between them, that in a letter to Bentley which has been quoted by Faraday in this place, he says :-

"It is inconceivable that inanimate brute matter should, without the mediation of something else, which is not material, operate upon and affect other matter without mutual contact, as it must do if gravitation, in the sense of Epicurus, be essential and inherent in it. . . . That gravity should be innate, inherent, and essential to matter, so that one body can act upon another at a distance, through a vacuum, without the mediation of anything else, by and through which their action and force may be conveyed from one to another, is to me so great an absurdity, that I believe no man who has in philosophical matters a competent faculty of thinking can ever fall into it."

Accordingly, we find in his "Optical Queries," and in his letters to Boyle, that Newton had very early made the attempt to account for gravitation by means of the pressure of a medium, and that the reason he did not publish these investigations "proceeded from hence only, that he found he was not able, from experiment and observation, to give a satisfactory account of this medium, and the manner of its operation in producing the chief phenomena of nature." *

The doctrine of direct action at a distance cannot claim for its author the discoverer of universal gravitation. It was first asserted by Roger Cotes, in his preface to the "Principia," which he edited during Newton's life. According to Cotes, it is by experience that we learn that all bodies gravitate. We do not learn in any other way that they are extended, nloveable, or solid. Gravitation, therefore, has as much right to be considered an essential property of matter as extension, mobility, or impenetrability.

And when the Newtonian philosophy gained ground in Europe, it was the opinion of Cotes rather than that of Newton that became most prevalent, till at last Boscovich propounded his theory, that matter is a congeries of mathematical points, each endowed with the power of attracting or repelling the others according to fixed laws. In his world, matter is unextended, and contact is impossible. He did not forget, however, to endow his mathematical points with inertia. In this some of the modern representatives of his school have thought that he "had not quite got so far as the strict modern view of 'matter' as being but an expression for modes or manifestations of "force." "+

But if we leave out of account for the present the development of the ideas of science, and confine our attention to the extension of its boundaries, we shall see that it was most essential that Newton's method should be extended to every branch of science to which it was applicable - that the forces with which bodies act on each other should be investigated in the first place, before attempting to explain how that force is transmitted. No men could be better fitted to apply themselves exclusively to the first part of the problem, than those who considered the second part quite unnecessary.

Accordingly Cavendish, Coulomb, and Poisson, the founders of the exact sciences of electricity and magnetism, paid no regard to those old notions of "magnetic effluvia " and " electric atmospheres," which had been put forth in the previous century, but turned their undivided attention to the determination of the law of force, according to which electrified and magnetised bodies attract or repel each other. In this way the true laws of these actions were discovered, and this was done by men who never doubted that the action took place at a distance, without the intervention of any medium, and who would have regarded the discovery of such a medium as complicating rather than as explaining the undoubted phenomena of attraction.

\section{(To be continued.)}

* Maclaurin's Account of Newton's Discoveries.

$\uparrow$ Review of Mrs. Somerville's "Molecular Science," Saturday Review', Feb. $x_{3}, x^{869}$. 